\title{
Step-wise learning of laparoscopic low anterior resection
}

\author{
Youn Young Park' ${ }^{1}$ Kang Young Lee ${ }^{2}$ \\ 'Department of Surgery, The Catholic University of Korea, Uijeongbu St. Mary's Hospital, Seoul, Korea \\ ${ }^{2}$ Department of Surgery, Severance Hospital, Yonsei University College of Medicine, Seoul, Korea
}

A primary goal of training is the development of one's own procedure by self-learning, which can be facilitated by laparoscopic surgery owing to its recordable system. This video demonstrates laparoscopic low anterior resection for a 56-year-old male diagnosed with synchronous S-colon cancer and rectal cancer. The surgery was performed by a second-year fellow in the colorectal division under supervision.

Keywords: Learning, Laparoscopy, Minimally invasive surgical procedures, Rectal neoplasms, Proctectomy

\author{
Received February 16, 2021 \\ Accepted March 5, 2021 \\ Corresponding author \\ Kang Young Lee \\ Department of Surgery, Severance \\ Hospital, Yonsei University College \\ of Medicine, 50-1 Yonsei-ro, \\ Seodaemun-gu, Seoul 03722, Korea \\ Tel: + 82-2-2228-2096 \\ Fax: +82-2-313-8289 \\ E-mail: KYLEE117@yuhs.ac \\ ORCID: \\ https://orcid.org/0000-0001-5944-2063
}

Copyright $\odot 2021$ The Journal of Minimally Invasive Surgery. All rights reserved.

This is an Open Access article distributed under the terms of the Creative Commons Attribution Non-Commercial License (http:// creativecommons.org/licenses/by-nc/4.0/) which permits unrestricted non-commercial use, distribution, and reproduction in any medium, provided the original work is properly cited.

\section{INTRODUCTION}

In the management of colorectal cancer, surgery is the mainstay of treatment. The oncologic principle that primary tumor and associated lymphatic drainage fields should be removed is the same as other malignancies. Total mesorectal excision (TME) is the key factor for rectal cancer surgery for adequate removal of tumor and regional lymph nodes [1]. Therefore, as a trainee to be a colorectal surgeon, it is mandatory to perform TME adequately. For this, one should understand the TME plane, the so-called holy plane, to mobilize the rectum along the correct surgical plane so that the oncologic and functional outcomes of a patient are not violated. Understanding multilaminar structures and interfascial spaces is essential. In that sense, minimally invasive surgery (MIS) can have advantages from an educational viewpoint. In the video clip, laparoscopic low anterior resection (LAR) is recorded. It was performed by a second-year fellow in the colorectal division within the training period.

\section{OPERATIVE PROCEDURES}

A 56-year-old man was diagnosed with synchronous S-colon cancer and mid-rectal cancer, and the clinical stage was T3NOM0 on initial computed tomography and pelvic magnetic resonance imaging. Laparoscopic LAR was performed for the patient. The procedures started with lateral to medial dissection. The relevant video clip is provided with this article (Supplementary Video 1).

\section{Lateral to medial dissection with apical node dissection}

The lateral dissection starts by detaching the natural adhesion of the sigmoid colon and proceeds to the splenic flexure. Toldt fascia separated from the mesocolon is observed, followed by the left gonadal vessel and the left ureter underneath the Toldt fascia, 
which are seen traversing the external left common iliac artery. This lateral dissection is the easiest step to develop the concept of multilaminar structures and interfascial space, by dividing the mesosigmoid and the Toldt fascia that are covered by shining lining at both sides. These linings continue down to the pelvic cavity. The lining covering the mesorectum is called the rectal proper fascia, and the lining covering the hypogastric nerves (HGN) is called the pre-HGN fascia, which is located between the presacral fascia and the rectal proper fascia [2].

After completing the lateral dissection, medial dissection starts with wide exposure of the mesosigmoid by grasping the inferior mesenteric artery (IMA) pedicle, and dissection progresses from the sacral promontory toward the root of the IMA. Care should be taken to avoid HGN injury during medial dissection at the sacral promontory level.

After making the peritoneal window between the lateral dissection and medial dissection planes, the apical node dissection starts from the anticipated point of the IMA bifurcation toward the IMA root. In this video, low ligation of the IMA with apical node dissection is performed. The inferior mesenteric vein (IMV) usually traverses the IMA bifurcation, which branches the superior rectal artery (SRA) and left colic artery (LCA). After skeletonization of the origin of the IMA for adequate apical lymphadenectomy with autonomic nerve preservation around the IMA root [3], the IMV and the SRA are ligated, preserving the LCA. During apical node dissection, care should be taken to avoid the superior hypogastric nerve plexus and preaortic nerve plexus.

\section{Pelvic dissection}

Pelvic dissection starts from the sacral promontory, where loose areolar tissues are abundant; thereby, the rectal proper fascia is easily dissected from the pre-HGN fascia, which overlies the HGN, preserving both HGNs. During the posterolateral part of the pelvic dissection, care should be taken to avoid injury of the pelvic splanchnic nerves and/or the inferior hypogastric nerve plexus, which runs downward tethering the rectal proper fascia. Sharp and meticulous dissection in front of the pre-HGN fascia can prevent injuries of autonomic nerves [2]. For further dissection, the mobilized rectum is hung by a tape to ensure adequate traction of the rectum. After making a U-shaped incision at the peritoneal reflection, anterior dissection proceeds, and the Denonvilliers' fascia can be seen. Upon further anterolateral dissection, care should be taken to avoid injury of the neurovascular bundles inserting into genitourinary organs that originate from the inferior hypogastric nerve plexus.

Developing the dissection plane in front of the pre-HGN fascia can lead the surgeon toward the proper avascular surgical plane, and not disrupt the parietal presacral fascia, so-called Waldeyer's fascia, preventing injury of the HGNs, which are located between the parietal presacral fascia and the pre-HGN fascia [2].

After completing mobilization of the rectum, the mesorectum is divided at the distal margin, and the rectum is resected with linear staplers. A Pfannenstiel incision is used for the mini-laparotomy site for specimen extraction. After the proximal colon is resected, end-to-end anastomosis is achieved by using a circular stapler under direct vision through the Pfannenstiel incision, and Lembert sutures are performed along the staple line.

\section{DISCUSSION}

As a trainee colorectal surgeon, one should thoroughly comprehend the TME plane for acceptable surgical and oncological outcomes. Appreciating the multilaminar structures and interfascial spaces is paramount. MIS has some advantages from an educational viewpoint in colorectal cancer surgery in this context. First, MIS can give a magnified view; therefore, a trainee may differentiate the fascial structures such as the rectal proper fascia, parietal presacral fascia, and pre-HGN fascia, relatively easier using the naked eye in the narrow and deep pelvic cavity. Second, it can be used for self-learning and validation by watching an expert's procedure during observational periods, followed by reviewing one's own procedures after recording. Step-wise learning of laparoscopic LAR can be initiated with the assistance of surgery to obtain an adequate concept for correct surgical planes. The correct surgical plane can then be identified by performing lateral dissection first, to apical node dissection with low ligation of the IMA, and to pelvic dissection, by a trainee after reviewing and self-validation of one's own surgical videos.

\section{NOTES}

\section{Ethical statements}

The Institutional Review Board of our institution approved this study with the waiver of informed consent (4-2020-1424), and we followed the principles of Declaration of Helsinki for health research ethics.

\section{Authors' contributions}

Conceptualization: All authors

Writing-original draft: YYP

Writing-review and editing: All authors

All authors read and approved the final manuscript.

\section{Conflict of interest}

All authors have no conflicts of interest to declare. 


\section{ORCID}

Youn Young Park, https://orcid.org/0000-0001-7724-1492

Kang Young Lee, https://orcid.org/0000-0001-5944-2063

\section{Supplementary materials}

Supplementary materials can be found via https://doi.org/10.7602/ jmis.2021.24.1.56.

\section{REFERENCES}

1. Dayal S, Battersby N, Cecil T. Evolution of surgical treatment for rectal cancer: a review. J Gastrointest Surg 2017;21:1166-1173.

2. Kinugasa Y, Murakami G, Suzuki D, Sugihara K. Histological identification of fascial structures posterolateral to the rectum. Br J Surg 2007;94:620-626.

3. Crocetti D, Cavallaro G, Tarallo MR, et al. Preservation of left colic artery with lymph node dissection of IMA root during laparoscopic surgery for rectosigmoid cancer. Results of a retrospective analysis. Clin Ter 2019;170:e124-e128. 